\title{
RESULTS ON SPLINE-FOURIER AND CIESIELSKI-FOURIER SERIES
}

\author{
FERENC WEISZ \\ Department of Numerical Analysis, Eötvös L. University \\ H-1117 Budapest, Pázmány P. sétány 1/C, Hungary \\ E-mail:weisz@ludens.elte.hu
}

Dedicated to Professor Zbigniew Ciesielski on his 70-th birthday

\begin{abstract}
Some recent results on spline-Fourier and Ciesielski-Fourier series are summarized. The convergence of spline Fourier series and the basis properties of the spline systems are considered. Some classical topics, that are well known for trigonometric and Walsh-Fourier series, are investigated for Ciesielski-Fourier series, such as inequalities for the Fourier coefficients, convergence a.e. and in norm, Fejér and $\theta$-summability, strong summability and multipliers. The connection between Fourier series and Hardy spaces is studied.
\end{abstract}

1. Introduction. It is known that the spline Fourier series of $f \in L_{p}$ converges a.e. and in $L_{p}$ norm to $f$, whenever $1 \leq p<\infty$. The maximal operator of the partial sums with respect to the spline (or unbounded Ciesielski) systems of order $(m, k)$ is bounded from the Hardy space $H_{p}$ to $L_{p}(1 /(m-k+2)<p<\infty)$ and is of weak type $(1,1)$. If $1<p<\infty$ then $H_{p}$ is equivalent to $L_{p}$. Moreover, the spline systems are unconditional and equivalent bases to the Haar system in $H_{p}(1 /(m-k+2)<p<\infty)$.

We investigate also the bounded Ciesielski systems, which can be obtained from the spline systems in the same way as the Walsh system from the Haar. Some results, that are well known for trigonometric and Walsh-Fourier series, are extended to CiesielskiFourier series. Paley and Hardy-Littlewood type inequalities are shown for CiesielskiFourier coefficients. If $f \in L_{p}(1<p<\infty)$ then the Ciesielski-Fourier series of $f$ converges

2000 Mathematics Subject Classification: Primary 41A15, 40F05, 42A24; Secondary 42C10, $42 \mathrm{~B} 08$.

Key words and phrases: Hardy spaces, $p$-atoms, spline and Ciesielski systems, Walsh system, Hardy-Littlewood and Paley type inequalities, Fejér means, strong summability, multipliers, Sunouchi operator.

This research was supported by Lise Meitner fellowship No M733-N04 and the Hungarian Scientific Research Funds (OTKA) No T043769, T047128, T047132.

The paper is in final form and no version of it will be published elsewhere. 
a.e. and in $L_{p}$-norm to $f$. It is proved that a lacunary sequence of the Ciesielski-Fourier series of $f \in H_{1}$ converges almost everywhere to $f$. The maximal operator of the Fejér means of the Ciesielski-Fourier series is bounded from $H_{p}$ to $L_{p}(1 / 2<p<\infty)$ and it is of weak type $(1,1)$. As a consequence we obtain that the Fejér means of the Ciesielski-Fourier series of a function $f \in L_{1}$ converges a.e. to $f$. Some other summability methods, called $\theta$-summability are considered as well. $\theta$-summability includes the well known summations of Weierstrass, Picar, Bessel, Riesz, de La Vallée-Poussin, Rogosinski and Riemann. The analogue of the strong convergence result is also given. It is proved that the maximal strong operator is of weak type $(1,1)$ and the Ciesielski-Fourier series of $f \in L_{1}$ is strong summable to $f$. Some multiplier theorems, e.g. the Marcinkiewicz multiplier theorem is shown for Ciesielski-Fourier series. The results can be extended to the multi-dimensional case, too.

2. Hardy spaces on the unit interval. We consider the unit interval $[0,1)$ with the Lebesgue measure $\lambda$. We briefly write $L_{p}$ instead of the real $L_{p}([0,1), \lambda)$ equipped with the norm (or quasinorm) $\|f\|_{p}:=\left(\int_{0}^{1}|f|^{p} d \lambda\right)^{1 / p}(0<p \leq \infty)$. The space $L_{p, \infty}=$ $L_{p, \infty}([0,1), \lambda)(0<p<\infty)$ consists of all measurable functions $f$ for which

$$
\|f\|_{p, \infty}:=\sup _{\rho>0} \rho \lambda(|f|>\rho)^{1 / p}<\infty
$$

while we set $L_{\infty, \infty}=L_{\infty}$. Note that $L_{p, \infty}$ is a quasi-normed space. It is easy to see that

$$
L_{p} \subset L_{p, \infty} \quad \text { and } \quad\|\cdot\|_{p, \infty} \leq\|\cdot\|_{p}
$$

for each $0<p \leq \infty$. The space $L_{p}\left(l_{r}\right)(1 \leq p, r<\infty)$ consists of all sequences $f:=$ $\left(f_{n}, n \in \mathbb{N}\right)$ of functions for which

$$
\|f\|_{L_{p}\left(l_{r}\right)}:=\left\|\left(\sum_{n \in \mathbb{N}}\left|f_{n}\right|^{r}\right)^{1 / r}\right\|_{p}<\infty .
$$

In order to have a common notation for the dyadic and classical Hardy spaces we define the Poisson kernels $P_{t}^{(m, k)}$. If $k \leq m$ then we introduce $P_{t}^{(m, k)}$ by

$$
P_{t}^{(m, k)}(x):=\frac{c t}{\left(t^{2}+|x|^{2}\right)} \quad(x \in \mathbb{R}, t>0) .
$$

If $k=m+1$ then we define $P_{t}^{(m, k)}$ as follows. For a fixed $t>0$, if $n \leq t<n+1$ for some $n \in \mathbb{N}$ then let

$$
P_{t}^{(m, k)}(x):=1_{\left[0,2^{-n}\right)}(x) \quad(x \in \mathbb{R}) .
$$

For a tempered distribution $f$ the non-tangential maximal function is defined by

$$
f_{*}^{(m, k)}(x):=\sup _{t>0}\left|\left(f * P_{t}^{(m, k)}\right)(x)\right| \quad(x \in \mathbb{R})
$$

where $*$ denotes the convolution.

For $0<p<\infty$ the Hardy space $H_{p}^{(m, k)}(\mathbb{R})$ consists of all tempered distributions $f$ for which

Now let

$$
\|f\|_{H_{p}^{(m, k)}(\mathbb{R})}:=\left\|f_{*}^{(m, k)}\right\|_{p}<\infty .
$$

$$
H_{p}:=H_{p}^{(m, k)}([0,1)):=\left\{f \in H_{p}^{(m, k)}(\mathbb{R}): \operatorname{supp} f \subset[0,1)\right\}
$$


Obviously, $H_{p}$ is the dyadic Hardy space if $k=m+1$. It is known (see Stein [56]) that the space $H_{p}$ coincides with $L_{p}$ if $1<p<\infty$.

The atomic decomposition is a basic tool in proving the results below. A function $a \in L_{\infty}$ is called a $p$-atom if there exists an interval $I \subset[0,1)$ such that

(i) supp $a \subset I$,

(ii) $\|a\|_{\infty} \leq|I|^{-1 / p}$,

(iii) $\int_{I} a(x) x^{j} d x=0$ where $j \in \mathbb{N}$ and $j \leq[1 / p-1]$.

Note that $[x]$ denotes the integer part of $x \in \mathbb{R}$.

In the dyadic case, i.e. if $k=m+1$, we consider only dyadic intervals $I$ and instead of (iii) we assume

(iii') $\int_{I} a(x) d x=0$.

The following result shows that for the boundedness of a sublinear operator $V$ from the Hardy space $H_{p}$ to $L_{p}$, it is enough to check the operator on $p$-atoms (Weisz [61]).

THEOREM 1. Suppose that the operator $V$ is sublinear and

$$
\int_{[0,1) \backslash 16 I}|V a|^{p} d \lambda \leq C_{p}
$$

for every p-atom a with support $I$, where $0<p \leq 1$. If $V$ is bounded from $L_{p_{1}}$ to $L_{p_{1}}$ for some $1<p_{1} \leq \infty$ then

$$
\|V f\|_{p} \leq C_{p}\|f\|_{H_{p}} \quad\left(f \in H_{p}\right) .
$$

3. Spline systems. We are going to introduce the spline systems as in Ciesielski [12]. Let us denote by $D$ the differentiation operator and define the integration operators

$$
G f(t):=\int_{0}^{t} f d \lambda, \quad H f(t):=\int_{t}^{1} f d \lambda .
$$

Define the $\chi_{n}, n=1,2, \ldots$, Haar system by $\chi_{1}:=1$ and

$$
\chi_{2^{n}+k}(x):= \begin{cases}2^{n / 2}, & \text { if } x \in\left((2 k-2) 2^{-n-1},(2 k-1) 2^{-n-1}\right), \\ -2^{n / 2}, & \text { if } x \in\left((2 k-1) 2^{-n-1},(2 k) 2^{-n-1}\right), \\ 0, & \text { otherwise, }\end{cases}
$$

for $n, k \in \mathbb{N}, 0<k \leq 2^{n}, x \in[0,1)$.

Let $m \geq-1$ be a fixed integer. Applying the Schmidt orthonormalization to the linearly independent functions

$$
1, t, \ldots, t^{m+1}, G^{m+1} \chi_{n}(t), \quad n \geq 2,
$$

we get the spline system $\left(f_{n}^{(m)}, n \geq-m\right)$ of order $m$. For $0 \leq k \leq m+1$ and $n \geq k-m$ define the splines

$$
f_{n}^{(m, k)}:=D^{k} f_{n}^{(m)}, \quad g_{n}^{(m, k)}:=H^{k} f_{n}^{(m)}
$$

of order $(m, k)$. Let us normalize these functions and introduce a more unified notation,

$$
h_{n}^{(m, k)}:= \begin{cases}f_{n}^{(m, k)}\left\|f_{n}^{(m, k)}\right\|_{2}^{-1} & \text { for } 0 \leq k \leq m+1 \\ g_{n}^{(m,-k)}\left\|f_{n}^{(m,-k)}\right\|_{2} & \text { for } 0 \leq-k \leq m+1 .\end{cases}
$$


We get the Haar system if $m=-1, k=0$ and the Franklin system if $m=0, k=0$. The systems $\left(h_{i}^{(m, k)}, i \geq|k|-m\right)$ and $\left(h_{j}^{(m,-k)}, j \geq|k|-m\right)$ are biorthogonal, i.e.

$$
\left(h_{i}^{(m, k)}, h_{j}^{(m,-k)}\right)= \begin{cases}1, & \text { if } i=j \\ 0, & \text { if } i \neq j,\end{cases}
$$

where $(f, g)$ denotes the usual scalar product $\int_{[0,1)} f g d \lambda$.

Ciesielski $[11,12]$ proved that the spline functions have the nice property

$$
\left|D^{N} h_{2^{\mu}+\nu}^{(m, k)}(t)\right| \leq C 2^{(N+1 / 2) \mu} q^{2^{\mu}\left|t-\nu / 2^{\mu}\right|},
$$

which is used several times in the proofs, where $m \geq-1,|k| \leq m+1, k+N \leq m+1$, $\mu \in \mathbb{N}$ and $\nu=1, \ldots, 2^{\mu}$.

In this paper the constants $C$ and $q$ depend only on $m$ and the constants $C_{p}$ depend only on $p$ and $m$. The symbols $C, q$ and $C_{p}$ may denote different constants in different contexts, however, $q$ denotes constants for which $0<q<1$.

3.1. Spline-Fourier series. The partial sums and the maximal operator of the partial sums of the spline-Fourier series are defined by

$$
P_{n}^{(m, k)} f:=\sum_{i=|k|-m}^{n}\left(f, h_{i}^{(m, k)}\right) h_{i}^{(m,-k)}=\int_{0}^{1} F_{n}^{(m, k)}(t, x) f(t) d t
$$

and

$$
P_{*}^{(m, k)} f:=\sup _{n \in \mathbb{N}}\left|P_{n}^{(m, k)} f\right|
$$

respectively, where $m \geq-1$ and $|k| \leq m+1$. Using (1), Domsta and Ciesielski [7] (see also Ciesielski [12]) proved that

$$
\left|F_{n}^{(m, k)}(t, x)\right|:=\left|\sum_{j=|k|-m}^{n} h_{j}^{(m, k)}(t) h_{j}^{(m,-k)}(x)\right| \leq C n q^{n|t-x|} .
$$

Since

$$
\int_{0}^{1}\left|F_{n}^{(m, k)}(t, x)\right| d t \leq C, \quad \int_{0}^{1}\left|F_{n}^{(m, k)}(t, x)\right| d x \leq C,
$$

we have

$$
\left\|P_{n}^{(m, k)} f\right\|_{p} \leq C_{p}\|f\|_{p} \quad\left(f \in L_{p}\right)
$$

for $p=\infty$ and $p=1$. By interpolation we get (3) for all $1<p<\infty$. Note that $C$ and $C_{p}$ are independent of $n$.

Corollary 1. Assume that $m \geq-1$ and $|k| \leq m+1$. If $f \in L_{p}$ for some $1 \leq p<\infty$ then $P_{n}^{(m, k)} f \rightarrow f$ in $L_{p}$ norm as $n \rightarrow \infty$.

This means that the spline systems are bases in $L_{p}(1 \leq p<\infty)$. There is also a vector valued version of (3) (see Weisz [69]).

Theorem 2. Assume that $m \geq-1,|k| \leq m+1$ and $f=\left(f_{i}, i \in \mathbb{N}\right) \in L_{p}\left(l_{r}\right)(1<p, r<$ $\infty)$. If $n(i)$ is an arbitrary natural number for each $i \in \mathbb{N}$ then

$$
\int_{0}^{1}\left(\sum_{i=0}^{\infty}\left|P_{n(i)}^{(m, k)} f_{i}\right|^{r}\right)^{p / r} d \lambda \leq C_{p, r} \int_{0}^{1}\left(\sum_{i=0}^{\infty}\left|f_{i}\right|^{r}\right)^{p / r} d \lambda .
$$


Ciesielski $[11,12,9]$ has generalized $(3)$ and showed that $P_{*}^{(m, k)}$ can be estimated by the Hardy-Littlewood maximal operator $M$. Hence $P_{*}^{(m, k)}$ is bounded on $L_{p}(1<p \leq \infty)$ and is of weak type $(1,1)$.

THEOREM 3. If $m \geq-1$ and $|k| \leq m+1$ then

$$
\left\|P_{*}^{(m, k)} f\right\|_{p} \leq C_{p}\|f\|_{p} \quad\left(f \in L_{p}\right)
$$

for all $1<p \leq \infty$. In particular, if $f \in L_{1}$ then

$$
\lambda\left(P_{*}^{(m, k)} f>\rho\right) \leq \frac{C}{\rho}\|f\|_{1} \quad(\rho>0) .
$$

The last weak type inequality and the usual density theorem due to Marcinkiewicz and Zygmund [29] imply

Corollary 2. If $m \geq-1,|k| \leq m+1$ and $f \in L_{1}$ then $P_{n}^{(m, k)} f \rightarrow f$ a.e. as $n \rightarrow \infty$.

Theorem 3 can be extended to Hardy spaces as follows (see Sjölin [55] and Weisz $[58,61]$, for $p=1$ Schipp and Simon [39]). Let

$$
p_{0}:= \begin{cases}1 /(m-k+2), & \text { if } k \leq m, \\ 0, & \text { if } k=m+1 .\end{cases}
$$

THEOREM 4. If $m \geq-1$ and $|k| \leq m+1$ then

$$
\left\|P_{*}^{(m, k)} f\right\|_{p} \leq C_{p}\|f\|_{H_{p}} \quad\left(f \in H_{p}\right)
$$

for all $p_{0}<p<\infty$. If $k \leq m$ then

$$
\left\|P_{*}^{(m, k)} f\right\|_{p_{0}, \infty} \leq C_{p_{0}}\|f\|_{H_{p_{0}}} \quad\left(f \in H_{p_{0}}\right) .
$$

3.2. Equivalent bases. The spline systems have very nice bases properties. To see this we will investigate the conditional partial sums and the square function of the spline-Fourier series:

$$
T_{n}^{(m, k)} f:=\sum_{i=|k|-m}^{n} \epsilon_{i}\left(f, h_{i}^{(m, k)}\right) h_{i}^{(m,-k)}
$$

and

$$
Q^{(m, k)} f:=\left(\sum_{i=|k|-m}^{\infty}\left|\left(f, h_{i}^{(m, k)}\right) h_{i}^{(m,-k)}\right|^{2}\right)^{1 / 2},
$$

respectively, where $m \geq-1,|k| \leq m+1$ and $\epsilon_{i}= \pm 1$. Of course, if each $\epsilon_{i}=1$ then $T_{n}^{(m, k)}=P_{n}^{(m, k)}$. Let $T^{(m, k)} f:=\lim _{n \rightarrow \infty} T_{n}^{(m, k)} f$, if the limit does exist.

Bočkariev [3] proved that the Franklin system is an unconditional basis in $L_{p}(1<$ $p<\infty)$. Some years later Ciesielski [12] verified that the spline systems are equivalent and unconditional bases in $L_{p}(1<p<\infty)$.

THEOREM 5. Assume that $m \geq-1,|k| \leq m+1$ and $1<p<\infty$. Then the systems $\left(h_{n}^{(m,-k)}\right)$ are unconditional bases in $L_{p}$ and

$$
\left\|T_{n}^{(m, k)} f\right\|_{p} \leq C_{p}\|f\|_{p} \quad\left(f \in L_{p}\right),
$$

uniformly in $n \in \mathbb{N}$. 
For $p=2$ see also Ropela [36]. Using this and Khinchine's inequality we can prove Theorem 6. If $m \geq-1,|k| \leq m+1,1<p<\infty$ and $f \in L_{p}$ then

$$
C_{p}^{-1}\|f\|_{p} \leq\left\|Q^{(m, k)} f\right\|_{p} \leq C_{p}\|f\|_{p} .
$$

Corollary 3. Assume that $m \geq-1,|k| \leq m+1$ and $1<p<\infty$. Then the Haar system $\left(\chi_{n}\right)$ and the spline system $\left(h_{n}^{(m,-k)}\right)$ are equivalent bases in the $L_{p}$ space, i.e. the following two series

$$
\sum_{n=1}^{\infty} a_{n} \chi_{n} \quad \text { and } \quad \sum_{n=|k|-m}^{\infty} a_{(n+m+1-|k|)} h_{n}^{(m,-k)}
$$

are equiconvergent in $L_{p}$.

The spline systems are also equivalent in $L_{p}\left(l_{r}\right)$ (see Figiel and Wojtaszczyk $[17,18]$ ). Actually, Figiel and Wojtaszczyk proved the equivalence in more general $U M D$ spaces.

Corollary 4. Assume that $m \geq-1,|k| \leq m+1$ and $1<p, r<\infty$. Then the Haar system $\left(\chi_{n}\right)$ and the spline system $\left(h_{n}^{(m,-k)}\right)$ are equivalent bases in the $L_{p}\left(l_{r}\right)$ space.

Maurey [31] proved that the classical $H_{1}$ space has an unconditional basis. His proof is non-constructive, he proved that $H_{1}$ is linearly isomorphic to the dyadic $H_{1}$ in which the Haar system is an unconditional basis. Carleson [5] constructed an unconditional basis in $H_{1}$ and Wojtaszczyk [71] verified that the Franklin system is an unconditional basis in $H_{1}$. Later Sjölin and Strömberg [52] and Wojtaszczyk [72] extended these results to $H_{p}$ spaces as follows. Let

$$
p_{1}:= \begin{cases}1 /(m-k+2), & \text { if } m \geq 0, \\ 0, & \text { if } m=-1 .\end{cases}
$$

THEOREM 7. If $m \geq-1,0 \leq k \leq m+1$ and $p_{1}<p<\infty$ then

$$
C_{p}^{-1}\|f\|_{H_{p}} \leq\left\|Q^{(m, k)} f\right\|_{p} \leq C_{p}\|f\|_{H_{p}} \quad\left(f \in H_{p}\right) .
$$

The next corollary follows easily from this.

Corollary 5. Assume that $m \geq-1,0 \leq k \leq m+1$ and $p_{1}<p<\infty$. Then the systems $\left(h_{n}^{(m,-k)}\right)$ are unconditional bases in $H_{p}$ and

$$
\left\|T_{n}^{(m, k)} f\right\|_{H_{p}} \leq C_{p}\|f\|_{H_{p}} \quad\left(f \in H_{p}\right),
$$

uniformly in $n \in \mathbb{N}$.

It is proved in Sjölin and Strömberg [53] and Wojtaszczyk [72] that the spline systems are not bases in $H_{p}$, if $p<p_{1}$. Moreover, the spline systems are not bases in the Hardy spaces if $k$ is negative (see Wojtaszczyk [72]).

The next result shows that the dyadic and classical Hardy spaces have equivalent bases (cf. Maurey [31]).

Corollary 6. Assume that $m \geq-1,0 \leq k \leq m+1$ and $p_{1}<p<\infty$. Then the Haar system $\left(\chi_{n}\right)$ and the spline system $\left(h_{n}^{(m,-k)}\right)$ are equivalent bases in the dyadic and classical $H_{p}$ spaces. 
The following result is due to Chang and Ciesielski [6] for $p=1$. For general $p$ see Weisz [58, 61].

Corollary 7. Assume that $0 \leq k \leq m+1$ and $p_{1}<p<\infty$. Then the following properties are equivalent:

(i) $f \in H_{p}$,

(ii) $Q^{(m, k)} f \in L_{p}$,

(iii) $\sup _{\epsilon}\left\|T^{(m, k)} f\right\|_{p}<\infty$,

(iv) $P_{n}^{(m, k)} f$ converges unconditionally in $L_{p}$.

Furthermore, the norms corresponding to (i), (ii) and (iii) are equivalent norms.

4. Ciesielski systems. First we define the Walsh system. Let

$$
r(x):= \begin{cases}1 & \text { if } x \in\left[0, \frac{1}{2}\right), \\ -1 & \text { if } x \in\left[\frac{1}{2}, 1\right),\end{cases}
$$

extended to $\mathbb{R}$ by periodicity of period 1 . The Rademacher system $\left(r_{n}, n \in \mathbb{N}\right)$ is defined by

$$
r_{n}(x):=r\left(2^{n} x\right) \quad(x \in[0,1), n \in \mathbb{N}) .
$$

The Walsh functions are given by

$$
w_{n}(x):=\prod_{k=0}^{\infty} r_{k}(x)^{n_{k}} \quad(x \in[0,1), n \in \mathbb{N})
$$

where $n=\sum_{k=0}^{\infty} n_{k} 2^{k},\left(n_{k}=0\right.$ or $\left.n_{k}=1\right)$.

Starting with the spline system $\left(h_{n}^{(m, k)}, n \geq|k|-m\right)$ we define the Ciesielski system $\left(c_{n}^{(m, k)}, n \geq|k|-m\right)$ in the same way as the Walsh system arises from the Haar system, namely,

$$
c_{n}^{(m, k)}:=h_{n}^{(m, k)} \quad(n=|k|-m, \ldots, 1)
$$

and

$$
c_{2^{\nu}+i}^{(m, k)}:=\sum_{j=1}^{2^{\nu}} A_{i, j}^{(\nu)} h_{2^{\nu}+j}^{(m, k)} \quad\left(1 \leq i \leq 2^{\nu}\right),
$$

where

$$
A_{i, j}^{(\nu)}=A_{j, i}^{(\nu)}=2^{-\nu / 2} w_{i-1}\left(\frac{2 j-1}{2^{\nu+1}}\right)
$$

(see Ciesielski [10], Ciesielski, Simon, Sjölin [8] or Schipp, Wade, Simon, Pál [40]). We get immediately that

$$
h_{2^{\nu}+j}^{(m, k)}:=\sum_{i=1}^{2^{\nu}} A_{i, j}^{(\nu)} c_{2^{\nu}+i}^{(m, k)} \quad\left(1 \leq j \leq 2^{\nu}\right) .
$$

As mentioned before,

$$
c_{n}^{(-1,0)}=w_{n-1} \quad(n \geq 1)
$$

is the usual Walsh system. The system $\left(c_{n}^{(m, k)}\right)$ is uniformly bounded and it is biorthogonal to $\left(c_{n}^{(m,-k)}\right)$ whenever $|k| \leq m+1$ (see Ciesielski [10]). 
4.1. Inequalities for Ciesielski-Fourier coefficients. In this subsection we assume that $k=0$ and use the notation $c_{n}^{(m, 0)}=c_{n}^{(m)}$. For simplicity we suppose here that $\left(f, c_{n}^{(m)}\right)=0$ for $-m \leq n \leq 1$. Of course, the theorems of this section can be shown without the last condition, too.

Paley [33] proved the inequality

$$
\left(\sum_{i=0}^{\infty}\left|\left(f, c_{2^{i}}^{(m)}\right)\right|^{2}\right)^{1 / 2} \leq C_{p}\|f\|_{H^{1}}
$$

for Walsh-Fourier coefficients (see also Coifman and Weiss [13]). A similar result was shown in the trigonometric case by Gundy and Varopoulos [20]. Recently the inequality was extended to unbounded Vilenkin systems and to all $p$ by Simon and Weisz [46] (see also Simon [47]). We generalized this inequality for Ciesielski-Fourier coefficients in [63].

TheOREM 8. Let $m \geq-1$ and $A_{n} \subset\left[2^{n}+1,2^{n+1}\right]$ be sets of integers. Then the following two conditions are equivalent.

(i) For all $f \in H_{p} \cap L_{1}$

$$
\left(\sum_{n=0}^{\infty} 2^{n(2-2 / p)} \sum_{k \in A_{n}}\left|\left(f, c_{k}^{(m)}\right)\right|^{2}\right)^{1 / 2} \leq C_{p}\|f\|_{H_{p}} \quad\left(p_{1}<p \leq 1\right),
$$

(ii) $\sup _{n \in \mathbb{N}}\left|A_{n}\right|<\infty$, where $\left|A_{n}\right|$ denotes the number of the elements of $A_{n}$.

By interpolation and duality argument we obtain a new version of Khinchine's inequality:

Corollary 8. If $m \geq-1,1<p \leq 2, A_{n} \subset\left[2^{n}+1,2^{n+1}\right]$ and $\sup _{n \in \mathbb{N}}\left|A_{n}\right|<\infty$ then

$$
\|f\|_{p} \sim\left(\sum_{n=0}^{\infty} \sum_{k \in A_{n}}\left|\left(f, c_{k}^{(m)}\right)\right|^{2}\right)^{1 / 2} \sim\|f\|_{B M O} \quad(0<p<\infty)
$$

for $f=\sum_{n=0}^{\infty} \sum_{k \in A_{n}}\left(f, c_{k}^{(m)}\right) c_{k}^{(m)}$, where BMO is the dual space of $H_{1}$.

Now we formulate the Hardy-Littlewood inequality for Ciesielski-Fourier coefficients (see Weisz [68]).

THEOREM 9. If $m \geq-1$ and $f \in H_{p} \cap L_{1}$ then

$$
\left(\sum_{n=2}^{\infty} \frac{\left|\left(f, c_{n}^{(m)}\right)\right|^{p}}{n^{2-p}}\right)^{1 / p} \leq C_{p}\|f\|_{H_{p}} \quad\left(p_{1}<p \leq 2\right) .
$$

This inequality is due to Hardy and Littlewood [22] for the trigonometric system (see also Coifman and Weiss [13]) and to Ladhawala [27] for the Walsh system. The inequality is also known for Fourier transforms (see Jawerth and Torchinsky [23]).

4.2. Convergence of Ciesielski-Fourier series. The partial sums and the maximal operators of the Ciesielski-Fourier series are defined by

$$
s_{n}^{(m, k)} f(x):=\sum_{i=|k|-m}^{n}\left(f, c_{i}^{(m, k)}\right) c_{i}^{(m,-k)}(x)=\int_{0}^{1} D_{n}^{(m, k)}(t, x) f(t) d t
$$


and

$$
s_{*}^{(m, k)} f:=\sup _{n \in \mathbb{N}}\left|s_{n}^{(m, k)} f\right|,
$$

respectively, where $m \geq-1$ and $|k| \leq m+1$. Here

$$
D_{n}^{(m, k)}(t, x):=\sum_{i=|k|-m}^{n} c_{i}^{(m, k)}(t) c_{i}^{(m,-k)}(x),
$$

is the $n$th Dirichlet kernel.

In contrast to the spline system, the Lebesgue functions are not bounded in this case. Ciesielski [10] proved that, similarly to the trigonometric and Walsh system,

$$
\int_{0}^{1}\left|D_{n}^{(m, k)}(t, x)\right| d t \leq C \log n \quad(n \in \mathbb{N}, x \in[0,1)) .
$$

The next sharper result, which is well known for the trigonometric, Walsh- and Vilenkin systems, is due to the author [66].

THEOREM 10. If $m \geq-1,|k| \leq m+1$ and $n \geq 0$ then

$$
\left|D_{n}^{(m, k)}(t, x)\right| \leq \frac{C}{|t-x|} \quad(t, x \in[0,1), t \neq x) .
$$

The following theorem was proved by Carleson [4] for the trigonometric system, by Billard [1] and Sjölin [54] (see also Schipp [44]) for the Walsh system and by Schipp [43] and Ciesielski $[11,12]$ for the Ciesielski system.

THEOREM 11. If $m \geq-1$ and $|k| \leq m+1$ then

$$
\left\|s_{*}^{(m, k)} f\right\|_{p} \leq C_{p}\|f\|_{p} \quad\left(f \in L_{p}\right)
$$

for all $1<p<\infty$.

This means that $s_{n}^{(m, k)}$ is uniformly bounded on $L_{p}(1<p<\infty)$. The result corresponding to Theorem 2 holds for Ciesielski systems as well ([69]).

TheOrem 12. Assume that $m \geq-1,|k| \leq m+1$ and $f=\left(f_{i}, i \in \mathbb{N}\right) \in L_{p}\left(l_{r}\right)(1<$ $p, r<\infty)$. If $n(i)$ is an arbitrary natural number for each $i \in \mathbb{N}$ then

$$
\int_{0}^{1}\left(\sum_{i=0}^{\infty}\left|s_{n(i)}^{(m, k)} f_{i}\right|^{r}\right)^{p / r} d \lambda \leq C_{p, r} \int_{0}^{1}\left(\sum_{i=0}^{\infty}\left|f_{i}\right|^{r}\right)^{p / r} d \lambda .
$$

This was proved by Marcinkiewicz and Zygmund for trigonometric Fourier series (see e.g. Zygmund [76, II. p. 225]) and by Sunouchi [57] for Walsh-Fourier series.

Theorem 11 imply

Corollary 9. Assume that $m \geq-1$ and $|k| \leq m+1$. If $f \in L_{p}$ for some $1<p<\infty$ then $s_{n}^{(m, k)} f \rightarrow f$ a.e. and in $L_{p}$ norm as $n \rightarrow \infty$.

Ciesielski, Simon and Sjölin [8] verified the a.e. convergence also for functions from $L(\log L)^{3}$.

Since the Ciesielski systems are uniformly bounded, due to a theorem of Bočkariev [2], this theorem does not hold for functions in $L_{1}$. Moreover, there is $f \in L_{1}$ such that the Ciesielski-Fourier series diverges a.e. (see Kazarian and Sargsian [24]). 
The convergence result is not true even for Hardy spaces. Ladhawala and Pankratz [26] verified for Walsh-Fourier series that there exists $f \in H_{1}$ such that the partial sums diverge everywhere (see also Schipp and Simon [37]). However, if $f \in H_{1}$ then a lacunary sequence of the partial sums converge a.e. to $f$. This was proved for Walsh-Fourier series by Ladhawala and Pankratz [26], for trigonometric Fourier series by Zygmund [76, II. p. 235] and for Vilenkin-Fourier series by Young [73]. Now we extend this result to Ciesielski-Fourier series (see Weisz [62]).

We say that a sequence $\left(n_{i}, i \in \mathbb{N}\right)$ of positive integers is lacunary if $n_{i+1} / n_{i}>\alpha>1$ for all $i \in \mathbb{N}$.

TheOrem 13. Assume that $m \geq-1,|k| \leq m+1$ and $\left(n_{i}, i \in \mathbb{N}\right)$ is a lacunary sequence of positive integers. If $f \in H_{1}$ then $\lim _{i \rightarrow \infty} s_{n_{i}}^{(m, k)} f=f$ a.e.

4.3. Fejér summability. Taking a suitable summability method, we can obtain a.e. convergence for $f \in L_{1}$ functions, too. It was proved by Lebesgue [28] that the Fejér means of the trigonometric Fourier series of an integrable function $f$ converge a.e. to $f$, i.e.

$$
\frac{1}{n} \sum_{k=0}^{n-1}\left(s_{k} f(x)-f(x)\right) \rightarrow 0 \quad \text { as } \quad n \rightarrow \infty
$$

for a.e. $x \in[-\pi, \pi]$, where $s_{k} f$ denotes the $k$ th partial sum of the Fourier series of $f$. Fejér summability for Walsh-Fourier series is due to Fine [19] (see also Schipp [42]).

The Fejér means and the maximal Fejér operator of the Ciesielski-Fourier series are given by

$$
\sigma_{n}^{(m, k)} f(x):=\frac{1}{n} \sum_{j=1}^{n} s_{j}^{(m, k)}(x)=\int_{0}^{1} K_{n}^{(m, k)}(t, x) f(t) d t
$$

and

$$
\sigma_{*}^{(m, k)} f:=\sup _{n \in \mathbb{N}}\left|\sigma_{n}^{(m, k)} f\right|,
$$

where $m \geq-1,|k| \leq m+1$ and

$$
K_{n}^{(m, k)}(t, x):=\frac{1}{n} \sum_{j=1}^{n} D_{j}^{(m, k)}(t, x)
$$

is the $n$th Fejér kernel. The $L_{1}$ norms of the Fejér kernels are bounded (see Weisz [59]), which implies the boundedness of $\sigma_{*}^{(m, k)}$ on $L_{\infty}$.

THEOREM 14. If $m \geq-1$ and $|k| \leq m+1$ then

$$
\int_{0}^{1}\left|K_{n}^{(m, k)}(t, x)\right| d t \leq C \quad(n \in \mathbb{N}, x \in[0,1)) .
$$

Using Theorem 1 we have proved in $[59,67]$ that $\sigma_{*}^{(m, k)}$ is bounded from the Hardy space $H_{p}$ to $L_{p}(1 / 2<p \leq \infty)$. From this it follows by interpolation that $\sigma_{*}^{(m, k)}$ is also of weak type $(1,1)$. More exactly, we have

THEOREM 15. If $m \geq-1$ and $|k| \leq m+1$ then

$$
\left\|\sigma_{*}^{(m, k)} f\right\|_{p} \leq C_{p}\|f\|_{H_{p}} \quad\left(f \in H_{p}\right)
$$


for all $1 / 2<p<\infty$. In particular, if $f \in L_{1}$ then

$$
\lambda\left(\sigma_{*}^{(m, k)} f>\rho\right) \leq \frac{C}{\rho}\|f\|_{1} \quad(\rho>0) .
$$

Moreover,

$$
\left\|\sigma_{*}^{(m, k)} f\right\|_{1 / 2, \infty} \leq C\|f\|_{H_{1 / 2}} \quad\left(f \in H_{1 / 2}\right) .
$$

The weak type $(1,1)$ inequality and the usual density theorem imply the analogue to (4):

Corollary 10. If $m \geq-1$ and $|k| \leq m+1$ then $f \in L_{1}$ implies

$$
\sigma_{n}^{(m, k)} f \rightarrow f \quad \text { a.e. as } n \rightarrow \infty .
$$

Simon [51] gave a counterexample which shows the following result for the WalshFourier series.

THEOREM 16. The operator $\sigma_{*}^{(-1,0)}$ is not bounded from $H_{p}$ to $L_{p}$, if $0<p<1 / 2$.

4.4. Other summability methods. It is easy to see that the Fejér means can also be given by

$$
\sigma_{n}^{(m, k)} f=\sum_{j=|k|-m}^{0}\left(f, c_{j}^{(m, k)}\right) c_{j}^{(m,-k)}+\sum_{j=1}^{n}\left(1-\frac{j-1}{n}\right)\left(f, c_{j}^{(m, k)}\right) c_{j}^{(m,-k)} .
$$

As a generalization of Fejér summability we introduce the $\theta$-summation. Assume that

$\theta \in L_{1}(\mathbb{R})$ is even and continuous, $\theta(0)=1$,

$\left(\theta\left(\frac{k}{n+1}\right)\right)_{k \in \mathbb{Z}} \in \ell_{1}, \lim _{x \rightarrow \infty} \theta(x)=0$

$\theta$ is twice continuously differentiable on $\mathbb{R}$ except at finitely many points,

$\theta^{\prime \prime} \neq 0$ except at finitely many points and finitely many intervals,

the left and right $\operatorname{limits}_{\lim _{x \rightarrow y \pm 0}} x \theta^{\prime}(x) \in \mathbb{R}$ exist at each point $y \in \mathbb{R}$, $\lim _{x \rightarrow \infty} x \theta^{\prime}(x)=0$.

Note that the second condition of $(5)$ is satisfied if $\theta$ is non-increasing on $(c, \infty)$ for some $c \geq 0$ or if it has compact support.

The $\theta$-means and the maximal $\theta$-operator of $f \in L_{1}$ are defined by

$$
\sigma_{n}^{(m, k), \theta} f(x):=\sum_{j=|k|-m}^{0}\left(f, c_{j}^{(m, k)}\right) c_{j}^{(m,-k)}+\sum_{j=1}^{n} \theta\left(\frac{j-1}{n}\right)\left(f, c_{j}^{(m, k)}\right) c_{j}^{(m,-k)}
$$

and

$$
\sigma_{*}^{(m, k), \theta} f:=\sup _{n \in \mathbb{N}}\left|\sigma_{n}^{(m, k), \theta} f\right| .
$$

Obviously, if $\theta(x):=(1-|x|) \vee 0$, then we get the Fejér means.

We have seen in [66] that if $\sigma_{*}^{(m, k)}$ is bounded on a quasi-normed space then so is $\sigma_{*}^{(m, k), \theta}$. Let $\mathbf{X}$ and $\mathbf{Y}$ be two complete quasi-normed spaces of measurable functions and $L_{\infty}$ be continuously and densely embedded into $\mathbf{X}$. Suppose that if $0 \leq f \leq g, f, g \in \mathbf{Y}$ then $\|f\|_{\mathbf{Y}} \leq\|g\|_{\mathbf{Y}}$. Moreover, if $f_{n}, f \in \mathbf{Y}, f_{n} \geq 0(n \in \mathbb{N})$ and $f_{n} \nearrow f$ a.e. as $n \rightarrow \infty$, then $\left\|f-f_{n}\right\|_{\mathbf{Y}} \rightarrow 0$. Note that the spaces $L_{p}$ and $L_{p, \infty}(0<p \leq \infty)$ satisfy these properties. 
Theorem 17. If (5) is satisfied and $\sigma_{*}^{(m, k)}: \mathbf{X} \rightarrow \mathbf{Y}$ is bounded, then $\sigma_{*}^{(m, k), \theta}$ is also bounded, i.e.,

$$
\left\|\sigma_{*}^{(m, k), \theta} f\right\|_{\mathbf{Y}} \leq C\|f\|_{\mathbf{X}} \quad(f \in \mathbf{X}) .
$$

The next result follows easily from Theorem 15 (see [66]).

THEOREM 18. If $m \geq-1,|k| \leq m+1$ and (5) is satisfied then

$$
\left\|\sigma_{*}^{(m, k), \theta} f\right\|_{p} \leq C_{p}\|f\|_{H_{p}} \quad\left(f \in H_{p}\right)
$$

for all $1 / 2<p \leq \infty$,

$$
\left\|\sigma_{*}^{(m, k), \theta} f\right\|_{1 / 2, \infty} \leq C\|f\|_{H_{1 / 2}} \quad\left(f \in H_{1 / 2}\right)
$$

and

$$
\sup _{\rho>0} \rho \lambda\left(\sigma_{*}^{(m, k), \theta} f>\rho\right) \leq C\|f\|_{1} \quad\left(f \in L_{1}\right) .
$$

Corollary 11. If $m \geq-1,|k| \leq m+1$ and (5) is satisfied then $f \in L_{1}$ implies

$$
\sigma_{n}^{(m, k), \theta} f \rightarrow f \quad \text { a.e. as } n \rightarrow \infty .
$$

One can show easily that $\theta$-summation includes Weierstrass, Picar, Bessel, Riesz, de La Vallée-Poussin, Rogosinski and Riemann summations. For the details see Weisz [66].

4.5. Strong summability. Taking absolute value in (4) we obtain the strong summability. More generally, we consider the convergence of the means

$$
\left(\frac{1}{n} \sum_{k=0}^{n-1}\left|s_{k} f(x)-f(x)\right|^{r}\right)^{1 / r} \text {. }
$$

Strong summability was considered first by Hardy and Littlewood [21] for trigonometric Fourier series. They verified that these means tend to 0 a.e. as $n \rightarrow \infty$, whenever $f \in L_{p}$ $(1<p<\infty)$. This result was generalized for $L_{1}$ functions and for $r=2$ by Marcinkiewicz [30] and for all $r>0$ by Zygmund [75].

For Walsh-Fourier series the strong summability was shown by Schipp [41, 45] for $r=2$ and by Rodin [35,34] for $r>0$ and for $B M O$ means. Now we formulate these results for Ciesielski-Fourier series.

Let

$$
S_{n}^{(m, k),(r)} f:=\left(\frac{1}{n} \sum_{j=1}^{n}\left|s_{j}^{(m, k)} f\right|^{r}\right)^{1 / r} \quad(n \in \mathbb{N})
$$

be the strong means and

$$
S_{*}^{(m, k),(r)} f:=\sup _{n \geq 1} S_{n}^{(m, k),(r)} f
$$

be the strong maximal operator, where $0<r<\infty$. In [64] we extended strong summability to Ciesielski-Fourier series.

THEOREM 19. If $m \geq-1,|k| \leq m+1$ and $0<r \leq 2$ then

$$
\left\|S_{*}^{(m, k),(r)} f\right\|_{\infty} \leq C\|f\|_{\infty} \quad\left(f \in L_{\infty}\right)
$$

and

$$
\sup _{\rho>0} \rho \lambda\left(S_{*}^{(m, k),(r)} f>\rho\right) \leq C\|f\|_{1} \quad\left(f \in L_{1}\right) .
$$


The next corollary follows easily by interpolation.

Corollary 12. If $m \geq-1,|k| \leq m+1,0<r \leq 2$ and $1<p<\infty$ then

$$
\left\|S_{*}^{(m, k),(r)} f\right\|_{p} \leq C_{p}\|f\|_{p} \quad\left(f \in L_{p}\right) .
$$

Note that the strong maximal operator is not bounded from $H_{1}$ to $L_{1}$ (see Schipp and Simon [38]). The weak type $(1,1)$ inequality in Theorem 17 and the usual density argument imply

Corollary 13. If $m \geq-1,|k| \leq m+1$ and $0<r \leq 2$ then $f \in L_{1}$ implies

$$
\left(\frac{1}{n} \sum_{j=1}^{n}\left|s_{j}^{(m, k)} f(x)-f(x)\right|^{r}\right)^{1 / r} \rightarrow 0 \quad \text { for a.e. } x \in[0,1) \text { as } n \rightarrow \infty .
$$

4.6. Multiplier theorems. For simplicity, in this subsection we suppose that

$$
\left(f, c_{j}^{(m, k)}\right)=0 \quad \text { for } \quad j=|k|-m, \ldots, 1 .
$$

The multiplier operator generalizes the operator $T_{n}^{(m, k)}$. For a given multiplier $\lambda=$ $\left(\lambda_{j}, j=2, \ldots\right)$ where the $\lambda_{j}$ 's are real numbers, the multiplier operators are defined by

$$
T_{\lambda}^{(m, k)} f:=\sum_{j=2}^{\infty} \lambda_{j}\left(f, c_{j}^{(m, k)}\right) c_{j}^{(m,-k)}
$$

if the sum exists and by

$$
T_{\lambda, n}^{(m, k)} f:=\sum_{j=2}^{n} \lambda_{j}\left(f, c_{j}^{(m, k)}\right) c_{j}^{(m,-k)} \quad(n \in \mathbb{N}),
$$

where $f \in L_{1}$.

The Marcinkiewicz multiplier theorem is generalized for Ciesielski systems in the next theorem (see Weisz [69]).

TheOREM 20. Assume that $m \geq-1,|k| \leq m+1$ and $f \in L_{p}(1<p<\infty)$. If

$$
\left|\lambda_{i}\right| \leq C, \quad \sum_{j=2^{i}+1}^{2^{i+1}-1}\left|\lambda_{j}-\lambda_{j+1}\right| \leq C \quad(i \in \mathbb{N})
$$

then $T_{\lambda}^{(m, k)} f \in L_{p}$ and

$$
\left\|T_{\lambda}^{(m, k)} f\right\|_{p} \leq C_{p}\|f\|_{p}
$$

The theorem can be proved by using Theorem 12 similarly to the trigonometric system (see Zygmund [76, II. p. 232]). The result for Vilenkin-Fourier series is due to Young [74].

The multiplier theorem is extended to Hardy spaces in [69]. Note that (6) follows from (7).

Theorem 21. Assume that $m \geq-1$ and $|k| \leq m+1$. If

$$
\left|\lambda_{n}\right| \leq C, \quad \sup _{2^{n}+1 \leq j \leq 2^{n+1}-1} j\left|\lambda_{j}-\lambda_{j+1}\right| \leq C \quad(n \in \mathbb{N})
$$


and

$$
\sum_{j=2^{n}+1}^{2^{n+1}-2} j\left|\lambda_{j}-2 \lambda_{j+1}+\lambda_{j+2}\right| \leq C \quad(n \in \mathbb{N})
$$

then, for $1 / 2<p<\infty$,

$$
\left\|\sup _{N \in \mathbb{N}}\left|T_{\lambda, 2^{N}}^{(m, k)} f\right|\right\|_{p} \leq C_{p}\|f\|_{H_{p}} \quad\left(f \in H_{p}\right) .
$$

Some other multiplier theorems for the Walsh system and for Hardy spaces can be found in Kitada [25], Onnewer and Quek [32], Simon [48, 49, 50] and Daly and Fridli $[14,16,15]$.

If the multiplier $\lambda$ is piecewise linear then we can prove a stronger result ([69]).

Theorem 22. Assume that $m \geq-1$ and $|k| \leq m+1$. If (7) is satisfied and

$$
\lambda_{j}-2 \lambda_{j+1}+\lambda_{j+2}=0 \quad \text { for all } j=2^{n}+1, \ldots, 2^{n+1}-2 \quad(n \in \mathbb{N})
$$

then (8) holds for all $p_{0}<p<\infty$.

For examples of multipliers we introduce

$$
\lambda_{j}^{(1)}:=\frac{j-1}{2^{n}} \quad \text { if } \quad 2^{n}+1 \leq j \leq 2^{n+1} \quad(n \in \mathbb{N})
$$

and

$$
\lambda_{j}^{(2)}:=\frac{2^{n}}{j-1} \quad \text { if } \quad 2^{n}+1 \leq j \leq 2^{n+1} \quad(n \in \mathbb{N}) .
$$

For more general multipliers we refer to [69]. It is easy to see that $\lambda^{(1)}$ satisfies the conditions of Theorems 21 and 22 and, moreover, $\lambda^{(2)}$ fulfills the conditions in Theorem 21. These two multipliers are used in [69] to prove some inequalities for the Sunouchi operators.

REMARK. For the multi-dimensional versions of the theorems presented in this paper see $[58,63,68,60,66,65,70,61]$. To shorten the paper we do not give these extensions here.

\section{References}

[1] P. Billard, Sur la convergence presque partout des séries de Fourier-Walsh des fonctions de l'espace $L^{2}[0,1]$, Studia Math. 28 (1967), 363-388.

[2] S. V. Bočkariev, Divergence on a set of positive measure of Fourier series for arbitrary bounded orthonormal set, Mat. Sb. 98 (1975), 436-449. (in Russian).

[3] S. V. Bočkariev, Some inequalities for Franklin series, Anal. Math. 1 (1975), 249-257.

[4] L. Carleson, On convergence and growth of partial sums of Fourier series, Acta Math. 116 (1966), 135-157.

[5] L. Carleson, An explicit unconditional basis in $H^{1}$, Bull. Sc. Math. 104 (1980), 405-416.

[6] S.-Y. A. Chang and Z. Ciesielski, Spline characterization of $H^{1}$, Studia Math. 75 (1983), 183-192.

[7] Z. Ciesielski and J. Domsta, Construction of an orthonormal basis in $C^{m}\left(I^{d}\right)$ and $W_{p}^{m}\left(I^{d}\right)$, Studia Math. 41 (1972), 211-224.

[8] Z. Ciesielski, P. Simon, and P. Sjölin, Equivalence of Haar and Franklin bases in $L_{p}$ spaces, Studia Math. 60 (1977), 195-210. 
[9] Z. Ciesielski, Properties of the orthonormal Franklin system, II, Studia Math. 27 (1966), 289-323.

[10] Z. Ciesielski, A bounded orthonormal system of polygonals, Studia Math. 31 (1968), 339346.

[11] Z. Ciesielski, Constructive function theory and spline systems, Studia Math. 53 (1975), 277-302.

[12] Z. Ciesielski, Equivalence, unconditionality and convergence a.e. of the spline bases in $L_{p}$ spaces, in: Approximation Theory, Banach Center Publications 4, PWN-Polish Scientific Publishers, Warsaw, 1979, 55-68.

[13] R. R. Coifman and G. Weiss, Extensions of Hardy spaces and their use in analysis, Bull. Amer. Math. Soc. 83 (1977), 569-645.

[14] J. E. Daly and S. Fridli, Walsh multipliers for dyadic Hardy spaces, Applic. Anal. 82 (2003), 689-700.

[15] J. E. Daly and S. Fridli, $H^{p}$ multipliers on the dyadic field, Ann. Univ. Sci. Budapest Eötvös Sect. Comput. 24 (2004), 275-284.

[16] J. E. Daly and S. Fridli, Translation invariant operators on Hardy spaces over Vilenkin groups, Acta Math. Acad. Paed. Nyíregyháziensis 20 (2004), 131-140.

[17] T. Figiel and P. Wojtaszczyk, Special bases in function spaces, in: W. B. Johnson and J. Lindenstrauss (eds.), Handbook of the Geometry of Banach Spaces, North-Holland, Amsterdam, 2001, 561-597, .

[18] T. Figiel, On equivalence of some bases to the Haar system in spaces of vector-valued functions, Bull. Polish Acad. Sci. Math. 36 (1988), 119-131.

[19] N. J. Fine, Cesàro summability of Walsh-Fourier series, Proc. Nat. Acad. Sci. USA 41 (1955), 558-591.

[20] R. F. Gundy and N. T. Varopoulos, A martingale that occurs in harmonic analysis, Ark. Mat. 14 (1976), 179-187.

[21] G. H. Hardy and J. E. Littlewood, Sur la série de Fourier d'une fonction à carré sommable, Comptes Rendus (Paris) 156 (1913), 1307-1309.

[22] G. H. Hardy and J. E. Littlewood, Some new properties of Fourier constants, J. London Math. Soc. 6 (1931), 3-9.

[23] B. Jawerth and A. Torchinsky, A note on real interpolation of Hardy spaces in the polydisk, Proc. Amer. Math. Soc. 96 (1986), 227-232.

[24] B. S. Kashin and A. A. Saakyan, Orthogonal Series, Transl. Math. Monographs 75, American Mathematical Society, Providence, 1989.

[25] T. Kitada, Weighted $H^{p}$ multipliers on locally compact Vilenkin groups, Monatsh. Math. 110 (1990), 283-295.

[26] N. R. Ladhawala and D. C. Pankratz, Almost everywhere convergence of Walsh Fourier series of $H^{1}$ functions, Studia Math. 59 (1976), 85-92.

[27] N. R. Ladhawala, Absolute summability of Walsh-Fourier series, Pacific J. Math. 65 (1976), 103-108.

[28] H. Lebesgue, Recherches sur la convergence des séries de Fourier, Math. Annalen 61 (1905), 251-280.

[29] J. Marcinkiewicz and A. Zygmund, On the summability of double Fourier series, Fund. Math. 32 (1939), 122-132.

[30] J. Marcinkiewicz, Sur la sommabilité forte des séries de Fourier, J. London Math. Soc. 14 (1939), 162-168.

[31] B. Maurey, Isomorphismes entre espaces $H_{1}$, Acta Math. 145 (1980), 79-120. 
[32] C. W. Onneweer and T. S. Quek, $H^{p}$ multiplier results on locally compact Vilenkin groups, Quart. J. Math. Oxford 40 (1989), 313-323.

[33] R. E. A. C. Paley, A remarkable system of orthogonal functions, Proc. Lond. Math. Soc. 34 (1932), 241-279.

[34] V. A. Rodin, The space BMO and strong means of Fourier series, Anal. Math. 16 (1990), 291-302.

[35] V. A. Rodin, The space BMO and strong means of Fourier-Walsh series, Math. USSR Sbornik 74 (1993), 203-218.

[36] S. Ropela, Spline bases in Besov spaces, Bull. Acad. Polon. Sci. 24 (1976), 319-325.

[37] F. Schipp and P. Simon, Remarks on a theorem of A.N. Kolmogorov, Matematikai Lapok 31 (1978-83), 117-123 (in Hungarian).

[38] F. Schipp and P. Simon, On some $\left(H, L_{1}\right)$-type maximal inequalities with respect to the Walsh-Paley system, in: Functions, Series, Operators, Proc. Conf. in Budapest, 1980, Coll. Math. Soc. J. Bolyai 35, North-Holland, Amsterdam, 1981, 1039-1045.

[39] F. Schipp and P. Simon, Investigation of Haar and Franklin series in Hardy spaces, Anal. Math. 8 (1982), 47-56.

[40] F. Schipp, W. R. Wade, P. Simon, and J. Pál, Walsh Series: An Introduction to Dyadic Harmonic Analysis, Adam Hilger, Bristol, New York, 1990.

[41] F. Schipp, Über die starke Summation von Walsh-Fourierreihen, Acta Sci. Math. (Szeged) 30 (1969), 77-87.

[42] F. Schipp, Über gewissen Maximaloperatoren, Ann. Univ. Sci. Budapest Sect. Math. 18 (1975), 189-195.

[43] F. Schipp, On a.e. convergence of expansion with respect to a bounded orthonormal system of polygonals, Studia Math. 58 (1976), 287-290.

[44] F. Schipp, Universal contractive projections and a.e. convergence, in: J. Galambos and I. Kátai (eds.), Probability Theory and Applications, Essays to the Memory of József Mogyoródi, Kluwer Academic Publishers, Dordrecht, 1992, 47-75.

[45] F. Schipp, On the strong summability of Walsh series, Publ. Math. Debrecen, 52 (1998), 611-633.

[46] P. Simon and F. Weisz, Paley type inequalities for Vilenkin-Fourier coefficients, Acta Sci. Math. (Szeged) 63 (1997), 107-124.

[47] P. Simon, A note on multipliers for Walsh system, Ann. Univ. Sci. Budapest Sect. Math. 40 (1997), 165-185.

[48] P. Simon, Hardy spaces and multipliers, Acta Sci. Math. (Szeged) 64 (1998), 183-200.

[49] P. Simon, Remarks on Walsh-Fourier multipliers, Publ. Math. Debrecen 52 (1998), 635657.

[50] P. Simon, Two-parameter multipliers on Hardy spaces, Colloq. Math. 77 (1998), 9-31.

[51] P. Simon, Cesàro summability with respect to two-parameter Walsh systems, Monatsh. Math. 131 (200), 321-334.

[52] P. Sjölin and J.-O. Strömberg, Basis properties of Hardy spaces, Ark. Mat. 21 (1983), 111-125.

[53] P. Sjölin and J.-O. Strömberg, Spline systems as bases in Hardy spaces, Israel J. Math. 45 (1983), 147-156.

[54] P. Sjölin, An inequality of Paley and convergence a.e. of Walsh-Fourier series, Arkiv för Math. 8 (1969), 551-570.

[55] P. Sjölin, Convergence almost everywhere of spline expansions in Hardy spaces, in: Topics in Modern Harmonic Analysis, Proc. Sem. Turin and Milan, 1982, 1983, 645-651. 
[56] E. M. Stein, Harmonic Analysis: Real-variable Methods, Orthogonality and Oscillatory Integrals, Princeton Univ. Press, Princeton, N.J., 1993.

[57] G.-I. Sunouchi, Strong summability of Walsh-Fourier series, Tohoku Math. J. 16 (1969), 228-237.

[58] F. Weisz, Multi-parameter unbounded Ciesielski systems and convergence results, East J. Approx. 6 (2000), 397-420.

[59] F. Weisz, On the Fejér means of the bounded Ciesielski systems, Studia Math. 146 (2001), $227-243$

[60] F. Weisz, Fejér summability of multi-parameter bounded Ciesielski systems, Analysis Math. 28 (2002), 135-155.

[61] F. Weisz, Summability of Multi-dimensional Fourier Series and Hardy Spaces, Kluwer, Dordrecht, 2002.

[62] F. Weisz, Almost everywhere convergence of Ciesielski-Fourier series of $H_{1}$ functions, Archiv der Math. 83 (2004), 135-145.

[63] F. Weisz, Paley type inequalities and multipliers for Ciesielski-Fourier series, Acta Sci. Math. (Szeged) 70 (2004), 77-89.

[64] F. Weisz, Strong summability of Ciesielski-Fourier series, Studia Math. 161 (2004), 269302.

[65] F. Weisz, Strong summability of more-dimensional Ciesielski-Fourier series, East J. Appr. 10 (2004), 333-354.

[66] F. Weisz, $\theta$-summability of Fourier series, Acta Math. Hungar. 103 (2004), 139-176.

[67] F. Weisz, Weak type inequalities for the Walsh and bounded Ciesielski systems, Analysis Math. 30 (2004), 147-160.

[68] F. Weisz, Hardy-Littlewood inequalities for Ciesielski-Fourier series, Analysis Math. 31 (2005), 217-233.

[69] F. Weisz, Marcinkiewicz multiplier theorem and the Sunouchi operator for CiesielskiFourier series, J. Appr. Theory 133 (2005), 195-220.

[70] F. Weisz, Multiplier theorems and strong summability for multi-dimensional CiesielskiFourier series, East J. Appr. (to appear).

[71] P. Wojtaszczyk The Franklin system is an unconditional basis in $H^{1}$, Ark. Mat. 20 (1982), 293-300.

[72] P. Wojtaszczyk, $H_{p}$-spaces, $p \leq 1$, and spline systems, Studia Math. 77 (1984), 289-320.

[73] W.-S. Young, Almost everywhere convergence of Vilenkin-Fourier series of $H^{1}$ functions, Proc. Amer. Math. Soc. 108 (1990), 433-441.

[74] W.-S. Young, Littlewood-Paley and multiplier theorems for Vilenkin-Fourier series, Can. J. Math. 46 (1994), 662-672.

[75] A. Zygmund, On the convergence and summability of power series on the circle II, Proc. London Math. Soc. 47 (1941), 326-350.

[76] A. Zygmund, Trigonometric Series, 3th edition, Cambridge Univ. Press, London, 2002. 